\title{
ORIGINAL ARTICLE Efficacy of HER2 retargeted herpes simplex virus as therapy for high-grade glioma in immunocompetent mice
}

\author{
E Reisoli ${ }^{1,6}$, E Gambini ${ }^{2,6}$, I Appolloni ${ }^{1}$, V Gatta ${ }^{3,4}$, M Barilari ${ }^{1}$, L Menotti ${ }^{4,5}$ and P Malatesta ${ }^{1,2}$
}

Replication-competent oncolytic herpes simplex viruses (HSVs) are considered a promising therapeutic approach for treatment of high-grade gliomas (HGGs), which are usually resistant to all the available treatments. We previously demonstrated that R-LM113, a recombinant HSV-1 fully retargeted to the human epidermal growth factor receptor 2 (HER2), is safe and prolongs survival of immunodeficient NOD/SCID mice in an intracranial model of HGG. However, because the treatment is designed to be employed on immunocompetent patients, it is necessary to test whether the host immune system impairs the viral efficacy or triggers a potentially fatal reaction. Here we confirmed the safety of R-LM113 in the immunocompetent mouse strain BALB/C, where it does not trigger encephalitis when intracranially inoculated. Then, we set up a syngeneic HGG model expressing HER2 in adult BALB/c mice and evaluated R-LM113 therapeutic efficacy. We found that R-LM113 leads to a significant improvement in animal survival when administered at the time of tumor inoculation, as well as when injected into an already established tumor. This study suggests that the host immune defenses do not curtail the oncolytic antitumor activity of replication-competent HSV R-LM113, which results effective in counteracting tumor growth.

Cancer Gene Therapy (2012) 19, 788-795; doi:10.1038/cgt.2012.62; published online 21 September 2012

Keywords: HSV; murine model; oncolytic virus; virotherapy

\section{INTRODUCTION}

High-grade gliomas (HGGs) are the most common malignant tumors originating from central nervous system and are among the most lethal form of cancer. Unfortunately, traditional therapies are often ineffective against HGG because of their highly invasive nature, combined with their chemo- and radio-resistance. Even with recent advances, the median survival of patients with grade IV gliomas is 15 months, with a 2 -year survival rate of about $26 \%{ }^{1}$

One promising alternative therapeutic approach, which has been explored in both preclinical and clinical trials, is the use of oncolytic viruses that provides a powerful mechanism of action compared with other therapies, because they can specifically kill tumor cells while sparing normal cells and they can also replicate and propagate within the tumor.

Engineered herpes simplex viruses (HSVs) have been among the most extensively studied oncolytic viruses against HGGs because of its several advantages: they do not integrate into host DNA, have a large genome, with up to $30 \mathrm{~kb}$ nonessential sequences amenable for removal and replacement with foreign sequences. From a safety standpoint, anti-herpetic drugs are readily available to abort a threatening infection, in a worst-case scenario. Moreover, thanks to its neurotropism, HSV is particularly suitable for an oncolytic therapy against $\mathrm{HGG}^{2-4}$

To date, oncolytic HSVs (oHSVs) have been applied in preclinical and clinical trials for a variety of tumors, including HGG demonstrating their safety and some degree of efficacy. ${ }^{2,5-8}$
We recently provided the first proof of principle of the safety and the efficacy of a replication-competent fully retargeted oHSV, R-LM113, in an immunodeficient (NOD/SCID) orthotopic model of HGG. ${ }^{9}$ R-LM113 is an engineered HSV, fully retargeted to the human epidermal growth factor receptor 2 (HER2). ${ }^{10}$ This receptor is well suited for this purpose because it is expressed in a considerable (15-80\%) fraction of $\mathrm{HGGs}^{11,12}$ and is a pejorative prognosis factor for several cancers including brain tumors. ${ }^{13-15}$ Notably, HER2 is not expressed in adult central nervous system; ${ }^{16}$ hence, it is a highly specific target for oncolytic virotherapy. The study was conducted in an immunodeficient mouse strain (NOD/ SCID) in order to better challenge the safety of the recombinant virus. Because of the extreme sensitivity of such strain, any residual tendency of the recombinant virus to spread in the healthy tissue would be easily recognized. However, for a real-life employment of the recombinant virus, it remained some concerns about its possible interactions with the immune system of the host. It is conceivable that the recombinant virus may evoke an immune response, triggering different unwanted reactions from fatal inflammation to a decrease of the oncolytic virus efficiency in terms of spread and cytotoxic effects. On the other hand, the host immune response against the virus may increase the efficacy in tumor clearance.

Here we focused our efforts on the development of an immunocompetent murine glioma model to evaluate whether the host immune response could affect the antitumor virotherapeutic effects. As mouse strains significantly diverge in their

${ }^{1}$ Department of Translational Oncology, IRCCS A.O.U. San Martino-IST, Genoa, Italy; ${ }^{2}$ Department of Experimental Medicine (DIMES), University of Genoa, Genoa, Italy; ${ }^{3}$ Department of Specialistic, Diagnostic and Experimental Medicine, University of Bologna, Bologna, Italy; ${ }^{4}$ Department of Experimental Pathology, Section of Microbiology and Virology, Alma Mater Studiorum-University of Bologna, Bologna, Italy and ${ }^{5}$ Department of Pharmacy and Biotechnology, University of Bologna, Bologna, Italy. Correspondence: Dr L Menotti, Department of Experimental Pathology, Section of Microbiology and Virology, Alma Mater Studiorum-University of Bologna, via S.Giacomo, 12, Bologna I-40126, Italy or Dr P Malatesta, Department of Translational Oncology, IRCCS A.O.U. San Martino-IST, Largo Rosanna Benzi 10, Genoa I-16132 Italy.

E-mail: laura.menotti@unibo.it or paolo.malatesta@unige.it

${ }^{6}$ These authors contributed equally to this work.

Received 12 July 2012; revised 27 August 2012; accepted 27 August 2012; published online 21 September 2012 
susceptibility to viral infections, we selected the BALB/c mouse strain, which is known to be susceptible to HSV. ${ }^{17,18}$

In this study, we provided evidence that both the safety and the therapeutic efficacy of the replication-competent HSV R-LM113 are not spoiled by the immune system.

\section{MATERIALS AND METHODS}

Animal procedures

Mice were handled in agreement with the guidelines conforming to current Italian regulations for the protection of animals used for scientific purposes (D.Ivo 27/01/1992, no. 116). Procedures were approved by the Ethical Committee for Animal Experimentation of the National Institute of Cancer Research and by the Italian Ministry of Health. The experiments were performed on the BALB/C mouse strain.

Intracranial injections into adult mouse brains were performed at bregma coordinates: anterior-posterior, $1.0 \mathrm{~mm}$ anterior; lateral, $1.5 \mathrm{~mm}$ left and $2.5 \mathrm{~mm}$ below the skull surface, as previously described. ${ }^{19}$ Animals were injected with up to $9 \mu \mathrm{l}$ of suspension, containing $2 \times 10^{4}$ to $7 \times 10^{4}$ cells or viral preparations, and then monitored daily for the appearance of neurological symptoms. Their brains were photographed on a Leica (Wetzlar, Germany) fluorescence stereo microscope.

Survival curves were determined using Kaplan-Meier analysis and survival between groups was compared by log-rank test.

\section{Cell cultures and transfection}

Platelet-derived growth factor B (PDGF-B)-induced brain tumors expressing DsRed fluorescent reporter were obtained as follows. Embryonic neural precursors were obtained from embryonic day 14 (E14) BALB/c mouse embryos as previously described. ${ }^{20}$ Cells were plated at a density of $3.6 \times 10^{5}$ cells per $\mathrm{cm}^{2}$ onto poly-D-lysine-coated (Sigma-Aldrich, Milano, Italy) 24-well plates in DMEM-F12 added with B27 supplement, human bFGF and EGF $\left(10 \mathrm{ng} \mathrm{ml}^{-1}\right)$. Immediately after plating, cells were transduced with retroviral vectors encoding PDGF-B and DsRed described previously. ${ }^{21,22}$ After 7 days, $2 \times 10^{4}$ transduced cells were intracranially inoculated in adult BALB/C mice.

Tumor cell cultures derived from PDGF-B/DsRed-induced gliomas (PDGFHGG cells) were maintained in DMEM-F12 added with B27 supplement, human bFGF and EGF (10 $\left.\mathrm{ng} \mathrm{ml}^{-1}\right)$ and plated on Matrigel (1:200, BD Biosciences, Milano, Italy). PDGF-HGG cells were transfected with the pcDNA3-HER2 plasmid ${ }^{23}$ by using Lipofectamine (Life Technologies Italia, Monza, Italy) and were selected with neomycin G418 (Life Technologies Italia).

\section{Immunostainings}

For histological analysis, brains were fixed with $4 \%$ paraformaldehyde, cryoprotected in $20 \%$ sucrose and sectioned with a Leica CM3050 S cryostat or paraffin-embedded and sectioned with a Leica RM2135 microtome. Immunostainings on brain sections or cultured cells were performed using the following antibodies: mouse monoclonal antibodies against HER2 (1:100; Santa Cruz Biotechnology, Santa Cruz, CA, USA), nestin (1:100; BD Biosciences); rabbit polyclonal antibodies against Olig2 (1:500; Sigma-Aldrich), Ng2 (1:200; Chemicon, Millipore, Milano, Italy) and human myeloperoxidase (MPO, 1:300; DakoCytomation, Milano, Italy); and rat antibodies against F4/80 (1:30, Serotec, Milano, Italy). Binding of primary antibodies was revealed with appropriate secondary anti-rabbit lgGDylight 488-conjugated (1:500; Jackson Immunoresearch, Milano, Italy), cy2conjugate anti-mouse IgG (1:100; Jackson Immunoresearch) and cy3conjugate anti-rat IgG (1:100; Jackson Immunoresearch). Nuclei were stained through 5 -min incubation in Hoechst 33342 solution $\left(1 \mu \mathrm{gl}^{-1}\right.$, Sigma-Aldrich).

\section{Cell infection}

The construction and production of the recombinant virus R-LM113, retargeted to HER2, and R-LM5, with wild-type tropism, have been described elsewhere. ${ }^{10}$

Cells were infected with R-LM5 or R-LM113, at the indicated multiplicity of infection estimated on the basis of titer determined in SK-OV-3 cells. The efficiency of infection was monitored by means of enhanced green fluorescent protein (EGFP) expression driven by both R-LM5 and R-LM113. The percentage of infected cells was evaluated by loading them on a hemocytometer and taking pictures/imaging with a motorized epifluorescence microscope (Axio Imager.M2, Zeiss, Oberkochen, Germany). Images were automatically analyzed with an ImageJ plug-in (Rasband, W.S., ImageJ; US National Institutes of Health, Bethesda, MD, http://rsb.info.nih.gov/ij/, 1997-2007), which allows to measure and plot area and fluorescence intensity of each cell. For mock treatment, R-LM113 was inactivated by exposure to UVC light source (G 30-W, Osram Sylvania; Danvers, MA, USA), emitting radiation of $253.7 \mathrm{~nm}$ for $20 \mathrm{~min}$.

\section{RESULTS}

Establishment of a BALB/c model of HGG expressing HER2

To study the effects of anti-HER2 oncolytic virotherapy on an immunocompetent system, we developed a murine HGG model in the $B A L B / c$ mouse strain, known to be among the most HSVsensitive mouse strains. ${ }^{24-26}$ We established the model by transducing BALB/C neural precursor cells explanted at embryonic day 14 (E14) with retroviruses encoding the growth factor PDGF-B, whose overexpression in neural progenitors is known to induce gliomas ${ }^{27,28}$ and the red fluorescence protein DsRed. Transduced neural precursor cells were subsequently transplanted in the brains of adult BALB/c mice where they generated primary tumors within about 100 days. Cells obtained from these primary tumors were cultured and engineered to express the human receptor HER2. Such cells (herein referred as to BALB/c-HGG) properly express HER2 on the cell membrane and closely resemble, in term of molecular marker expression and tumorigenic potential, C57BL/ 6-HGG cells expressing HER2 as previously described (Figures 1a and e). ${ }^{9}$ By immunocytochemical analysis, we showed that both BALB/c-HGG-HER2 and C57BL/6-HGG-HER2 cells display markers typical of oligodendroglial precursors, such as Nestin, Ng2 and Olig2 (Figures $1 b-d$ and $f-h$ ). Moreover, animals intracranially transplanted with HER2-positive BALB/c-HGG die with a median survival of $47 \pm 1$ days, similar to that of NOD/SCID mice transplanted with C57BL/6-HGG-HER2 (51 \pm 2 days) cells, due to the formation of large DsRed-positive tumor masses (Figures $1 \mathrm{i}$ and k). HGGs derived from BALB/c and C57BL/6-HGG-HER2 cells histologically closely resemble each other and human HGGs because they are characterized by a rather compact structure and wide necrotic areas surrounded by highly proliferating cells forming pseudopalisades (Figures $1 \mathrm{j}$ and $\mathrm{I}$ ).

The $B A L B / c$ immunocompetent mouse strain is sensitive to HSV infection

First, we confirmed BALB/C susceptibility to HSV infection by intracranially inoculating in adult BALB/c mice, increasing doses of R-LM5, a recombinant HSV exhibiting wild-type tropism, and expressing EGFP reporter. In this assay, all the three mice inoculated with $10^{6}$ plaque-forming units developed lethal encephalitis within 6 days. At death, the explanted brain revealed wide EGFP-positive areas, ascribable to virus spread (Figure 2a). Histology of brain sections was consistent with encephalitis, showing broad areas of extravasation and blood-vessel wall thickening (Figure 2b). Furthermore, massive infiltration of granulocytes and macrophages was detectable, thanks to myeloperoxidase and F4/80 stainings (Figure 2c). On the contrary, none of the animals intracranially inoculated with $10^{6}$ plaque-forming unit of the fully retargeted recombinant R-LM113 exhibited neurological symptoms within the time frame of observation ( 7 days), and the histological analysis did not show any sign of viral neurotoxicity or inflammation (Figures $2 \mathrm{e}$ and $\mathrm{f}$ ).

We then demonstrated that BALB/C-HGG-HER2 cells are susceptible to R-LM113 infection and spread, in vitro, by exposing replicate cultures of HER2-negative and HER2-positive BALB/cHGG cells to serial dilutions of R-LM113 or R-LM5, as control. After $24 \mathrm{~h}$, infection was detected as expression of the reporter gene EGFP encoded by the virus (Figures $3 a-d^{\prime}$ ). These data further confirm the full retargeting of R-LM113, because the recombinant HSV was not able to enter HER2-negative BALB/c-HGG cells. 

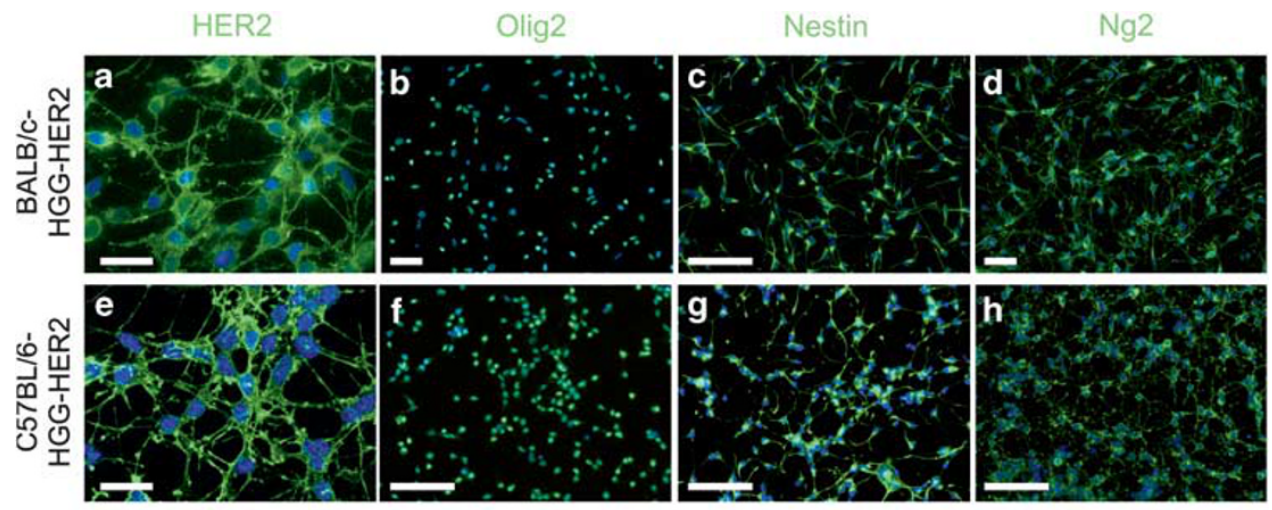

BALB/C

NOD/SCID
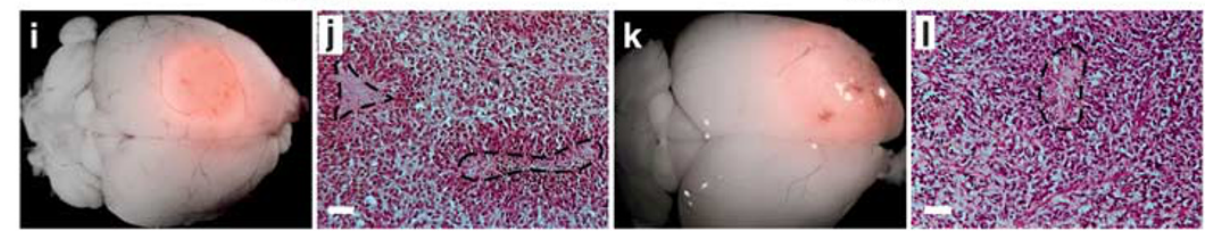

Figure 1. Establishment of a PDGF-induced glioma model expressing HER2 in the BALB/c mouse strain. (a-h) Immunofluorescence stainings of BALB/c-HGG-HER2 (a-d) and C57BL/6-HGG-HER2 (e-h) cultured cells for the indicated markers in green. Nuclei are counterstained in blue with Hoechst 33342. (i, k) Merged fluorescence and brightfield images of typical BALB/c-HGG-HER2- (i) and NOD/SCID-HGG-HER2-derived (k) tumors in dorsal view. The tumors are detectable as DsRed expression. (j, l) Coronal sections of BALB/c-HGG-HER2 (j) and NOD/SCID-HGGHER2 (I) stained with hematoxylin and eosin. The dashed lines define the contour of pseudopalisades. Scale bars: $25 \mu \mathrm{m}(\mathbf{a}, \mathbf{e}) ; 100 \mu \mathrm{m}(\mathbf{b}, \mathbf{d}$, $\mathbf{f}-\mathbf{h}, \mathbf{j}, \mathrm{I})$.

Infection efficiency of R-LM113 in BALB/c-HGG-HER2 cells was compared with that of the wild-type tropism HSV R-LM5 by infecting BALB/C-HGG-HER2 cells at the same multiplicity of infection of either virus (that is, 1 plaque-forming unit per cell, as determined from titration in SK-OV-3 cells) and by evaluating the number of infected cells after $24 \mathrm{~h}$. In these experiments, R-LM113 infected about $6 \%$ of BALB/c-HGG-HER2 cells (Figure 3e), whereas R-LM5 infected about $60 \%$ of cells (Figure 3f). Thus, the infection efficiency of R-LM113 in BALB/C-HGG-HER2 cells was about one order of magnitude lower than that of R-LM5, in line with the difference we showed for C57BL/6-HGG-HER2. ${ }^{9}$ The susceptibility of BALB/c-HGG-HER2 cells to R-LM113 was, however, lower than that of C57BL/6-HGG-HER2 cells, likely because of their three times lower level of HER2 (Supplementary Figure 1). Finally, R-LM113 ability to spread in BALB/c-HGG-HER2 cells was demonstrated by infecting such cells with R-LM113 at low multiplicity of infection (0.025) and by monitoring the formation of plaques during the time (Figures $3 \mathrm{~g}-\mathrm{i}$ ).

Oncolytic efficacy of R-LM113 in an immunocompetent environment

We then investigated the therapeutic efficacy of R-LM113, following two experimental schemes differing on the timing of viral treatment.

In the first approach, representing the earliest possible treatment, the virus was put in contact with the tumor cells at the same time of intracranial transplantation. We therefore intracranially transplanted, in three independent sessions, a pool of 35 adult BALB/C mice with $1 \times 10^{4}$ to $6 \times 10^{4}$ BALB/c-HGG-HER2 cells. Seventeen mice (herein referred to as 'R-LM113 earlytreatment set') received, in addition and at the same time, between $5 \times 10^{3}$ and $1 \times 10^{4}$ BALB/c-HGG-HER2 cells that were infected in vitro $48 \mathrm{~h}$ before with R-LM113 at a multiplicity of infection between 5 and 10 . The remaining 18 mice were used as control and herein referred to as 'early-treatment control set'. Mice were then monitored for 160 days.
Survival time of mice belonging to the R-LM113 early-treatment set appeared significantly increased as compared with that of the control set, showing a median survival time of 77 versus 46 days, respectively, (log-rank test $P<5 \times 10^{-4} ;$ Figure 4 a). All the animals of the control set exhibited neurological symptoms within 81 days after transplantation, because of the presence of DsRed-positive large tumor masses (Figure 4b). On the contrary, $>40 \%$ of the R-LM113 early-treatment set did not show any sign of distress in the same time frame. The brains of the animals belonging to the R-LM113 early-treatment set were examined when they showed the first neurological symptoms and almost all of them showed the presence of DsRed-positive tumor masses, sometime scattered with EGFP-positive areas, indicating an ongoing infection of R-LM113 (Figure 4c). Two mice belonging to the R-LM113 earlytreatment set survived until the end of the planned observation period (160 days) and were killed. One of them bore a DsRedpositive tumor mass harboring an EGFP-positive area, whereas the other one appeared tumor-free.

In the second approach, the treatment with oncolytic virus was performed at later stage (that is, 26 days after transplantation of $2 \times 10^{4}$ BALB/c-HGG-HER2 cells), on an already established tumor, to mimic a possible therapeutic application of the oncolytic virus. The treatment consisted in a single intratumor injection of $5.5 \times 10^{5}$ plaque-forming unit of R-LM113 in 19 adult BALB/C mice (herein referred to as 'R-LM113 late-treatment set'). In parallel, as control, 18 mice were injected with the same volume of ultraviolet-inactivated viral preparation (herein referred to as 'UVR-LM113 late-treatment control set'). We took into account only the animals surviving at least 5 days after virus injection (16 in R-LM113 late-treatment set and 14 for the control), a time likely sufficient for spread of the virus, and excluded mice that did not recover from the intracranial stereotaxic injection.

Mice belonging to the R-LM113 late-treatment set showed a significant increase of the median survival compared with that of the control set $(P<0.005$; Figure $4 d)$.

Although all the mice of the R-LM113 late-treatment set died due to the formation of DsRed-positive tumor mass, 6 out of 16 
R-LM5
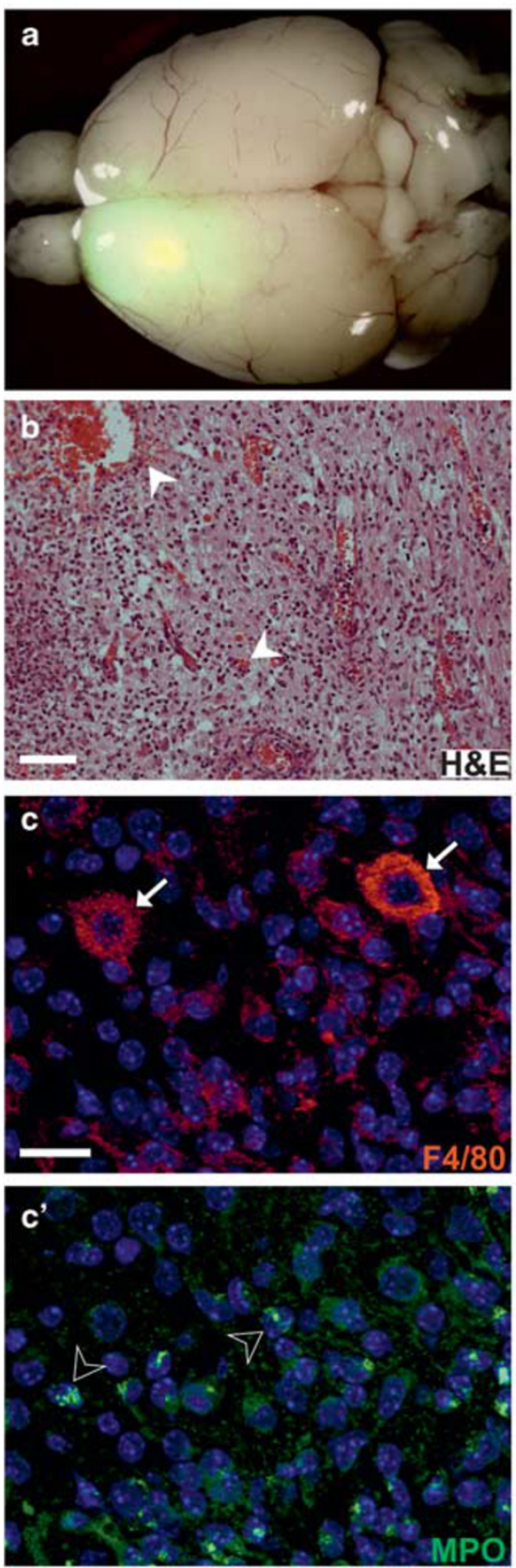

R-LM113
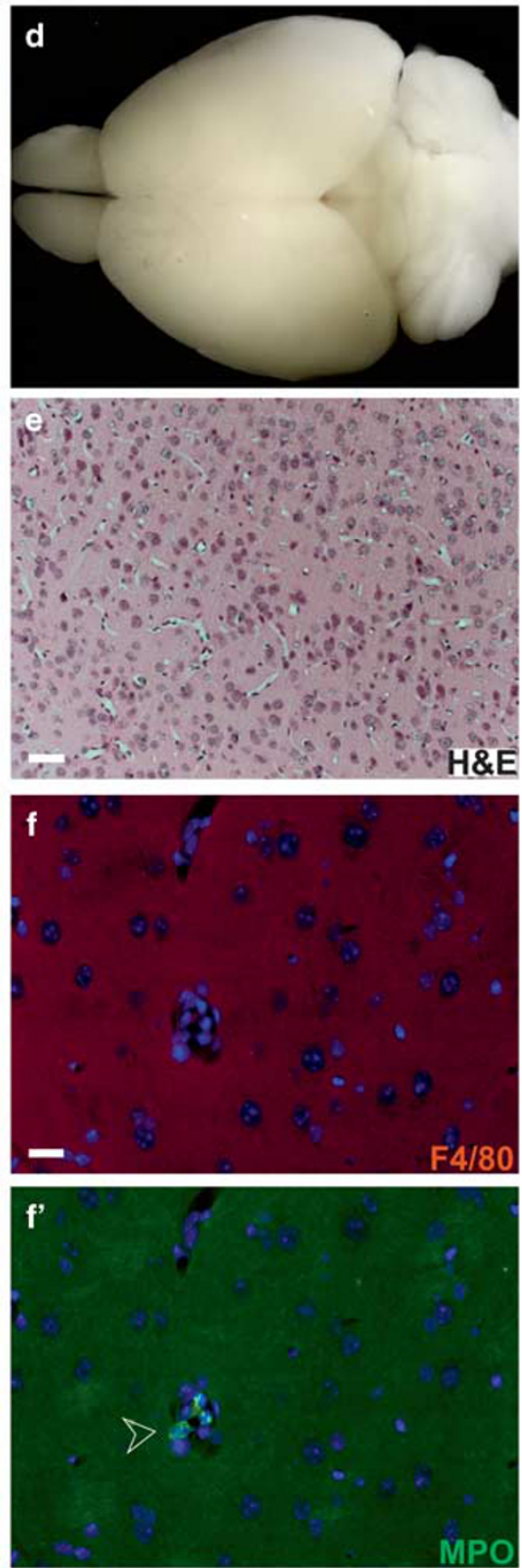

Figure 2. BALB/C mice are sensitive to HSV infection but the retargeted HSV R-LM113 is safe in vivo. Merged fluorescence and brightfield images of adult BALB/c mouse brains after injection of $10^{6}$ plaque-forming unit of R-LM5 (a) or R-LM113 (d). Viral spread is visualized as EGFP expression. Coronal sections of adult BALB/C mouse brains after injection of R-LM5 (b, c) or R-LM113 (e, f), stained with hematoxylin and eosin $(\mathbf{b}, \mathbf{e})$ or with antibody for the indicated markers $\left(\mathbf{c}, \mathbf{c}^{\prime}, \mathbf{f}, \mathbf{f}^{\prime}\right)$. To note, the presence of extravasation areas (arrowheads in $\left.\mathbf{b}\right)$ and infiltration of macrophages (arrows in c) and granulocytes (hollow arrowheads in $\mathbf{c}^{\prime}$ ) in the parenchyma of R-LM5-injected brains. On the contrary, granulocytes are exclusively found inside blood vessels of R-LM113-injected brains (hollow arrowhead in $\mathbf{f}^{\prime}$ ). Brains were harvested 6 days after injection with R-LM5 or 7 days after injection with R-LM113. Scale bars: $50 \mu \mathrm{m}$ (b, e); $20 \mu \mathrm{m}$ (c, f).

displayed the presence of EGFP-positive areas (Figure 4e), immunopositive for a polyclonal antiserum against the HSV glycoprotein gM (Supplementary Figure 2), demonstrating that the virus was spreading into the tumor.

\section{DISCUSSION}

In this study, we provide evidence for the antitumor efficacy of the recombinant oHSV R-LM113 in an immunocompetent animal model of HGG.
We previously showed R-LM113 safety and efficacy in counteracting HGG growth in the immunocompromised NOD/SCID mouse strain. ${ }^{9}$ Nevertheless, although the brain is an immunoprivileged location, a therapeutic strategy based on oncolytic viruses should take into account possible interaction between the viruses and the host immune system. Orthotopic cancer models in immunocompetent animals susceptible to the oncolytic virus represent the most reliable and predictive preclinical model for oncolytic virotherapy. ${ }^{29}$

An immune response could, in fact, limit viral spread, hence, causing a decrease of cytotoxic effects as it has been shown for 

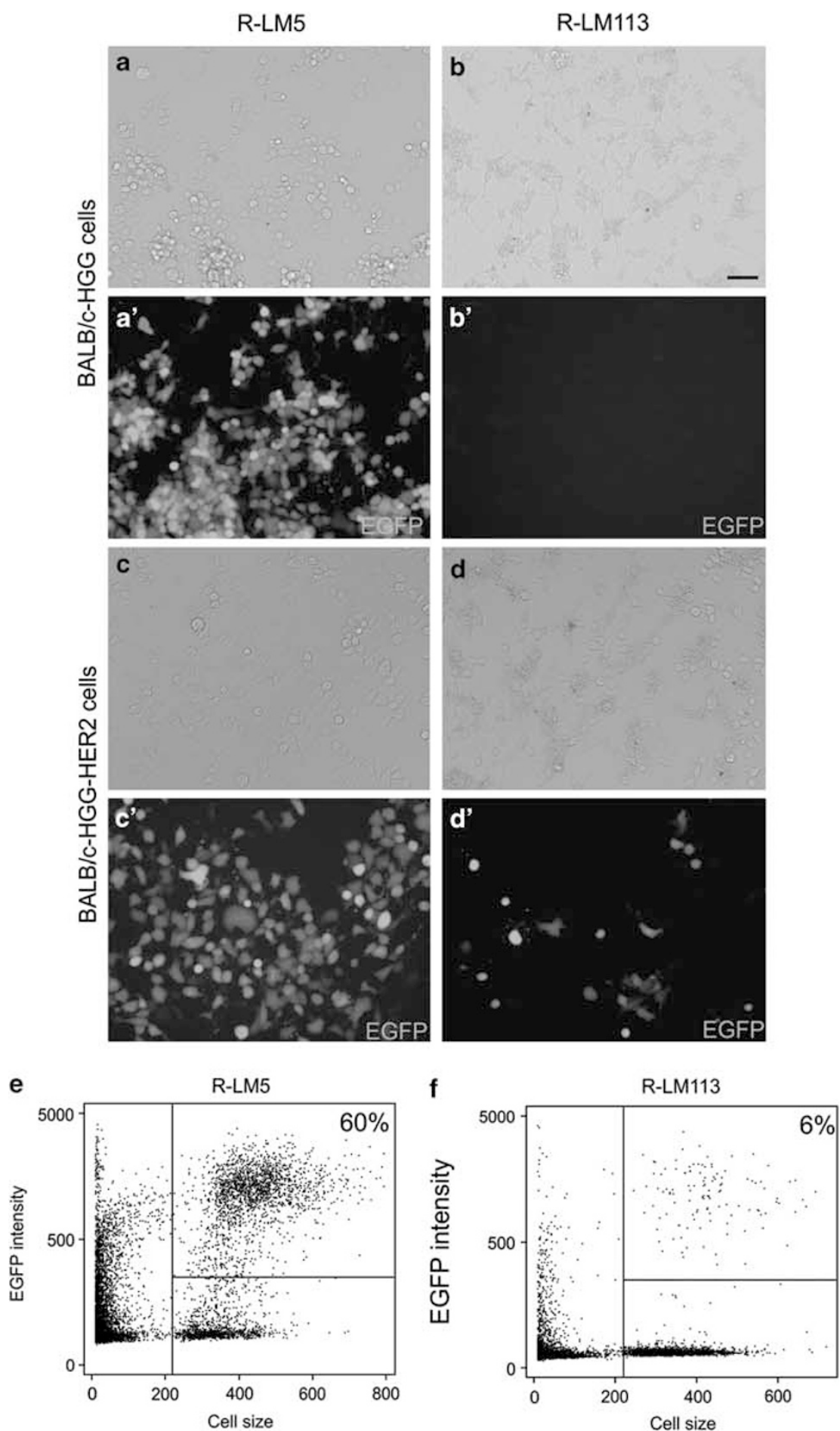

R-LM113 spreading in BALB/C-HGG-HER2 cells
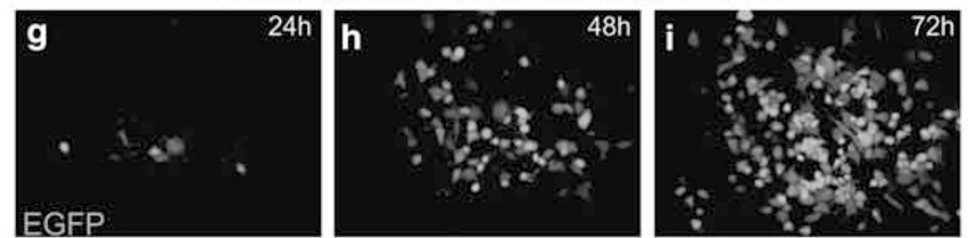

Figure 3. BALB/c-HGG-HER2 cells are permissive to R-LM113 infection. (a-d') Matched micrographs showing brightfield (a-d) and virus encoded EGFP fluorescence $\left(\mathbf{a}^{\prime}-\mathbf{d}^{\prime}\right)$ of BALB/c-HGG $(\mathbf{a}, \mathbf{b})$ or BALB/c-HGG-HER2 cells $(\mathbf{c}, \mathbf{d}) 24 \mathrm{~h}$ after exposure to R-LM5 (a, c) or R-LM113 (b, d) at multiplicity of infection $(\mathrm{MOI})=1$. Dot plots of BALB/c-HGG-HER2 cells $24 \mathrm{~h}$ after being exposed to R-LM5 (e) or R-LM113 (f). Numbers in quadrants indicate the percentage of infected cells evaluated as EGFP expression. Lines correspond to the thresholds used. (g-i) Cell-to-cell spread of the R-LM113 recombinant virus in BALB/c-HGG-HER2 cells at different time points after infection with $\mathrm{MOI}=0.2$. Scale bar: $50 \mu \mathrm{m}$. 

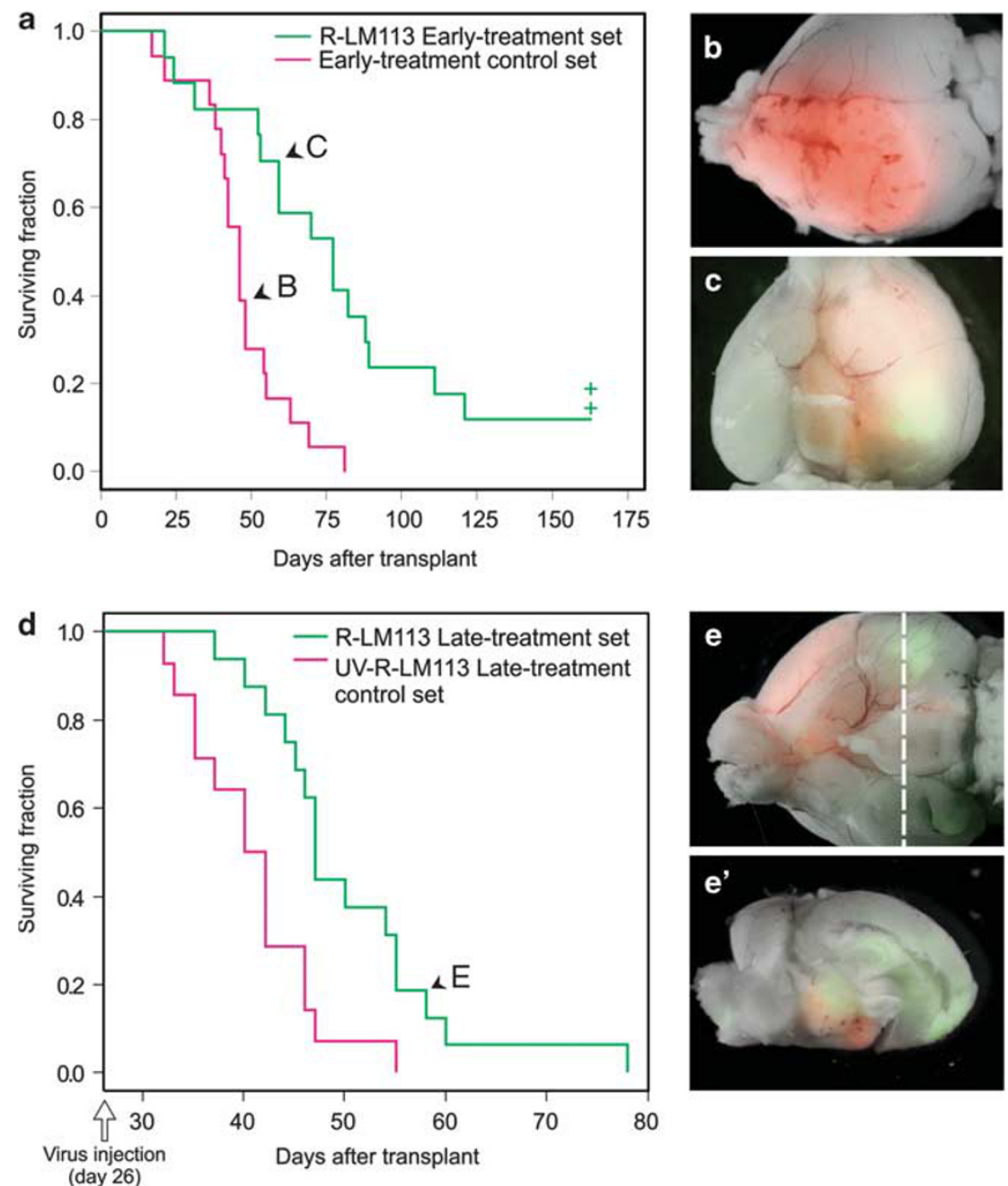

(day 26)

Figure 4. R-LM113 is effective in counteracting glioma growth in the immunocompetent BALB/c mouse strain. (a) Kaplan-Meier plot for $\mathrm{BALB} / \mathrm{c}$ animals transplanted with BALB/c-HGG-HER2 cells alone (red line, early-treatment control set) or together with R-LM113 infected BALB/c-HGG-HER2 (green line, R-LM113 early-treatment set). (b, c) Merged DsRed and EGFP fluorescence and brightfield images of brain from a mouse of the control set, in dorsal view (b, arrowhead labeled as B in $\mathbf{a}$ ) and from one of the R-LM113 early-treatment set, in ventral view (c, arrowhead labeled as C in a). To note, the presence of an EGFP-positive region in the R-LM113-treated brain. (d) Kaplan-Meier plot for BALB/c animals bearing BALB/C-HGG-HER2 tumors inoculated with R-LM113 (green line, median survival 21 days) or with ultraviolet-inactivated R-LM113 (red line, median survival 16 days). (e) Merged red and green fluorescence and brightfield images of a brain from a mouse of the R-LM113 late-treatment set died at 32 days (e, arrowhead labeled as E in panel d) following R-LM113 inoculation. (e') Coronal section along the dashed line of the brain in panel e; in red is shown DsRed expressed by cells of the tumor, in green EGFP expressed from the virus.

adenoviral vectors. ${ }^{30,31}$ Besides, a very strong immune response could hypothetically trigger a fatal inflammation leading to death. On the other hand, it has been demonstrated that the oncolytic efficacy of an attenuated HSV vector may be increased by the virally induced antitumor immunity, ${ }^{32}$ either locally by the recruitment of immune effector cells, which may increase oncolytic activity and improve tumor clearance, or at distant sites by cross-priming the immune response to the tumor. ${ }^{33}$ With this respect, in some instances HSVs have been armed in order to achieve a better immune-mediated tumor killing. ${ }^{34,35}$

Syngeneic models for gliomas have been developed in rats and mice. ${ }^{36}$ The oncolytic virotherapy approaches undertaken with adenoviruses exploited mostly replication-deficient, locally delivered vectors carrying immunomodulatory molecules to boost the immune response and to recruit bone marrowderived dendritic cells, ${ }^{37,38}$ clearly bypassing the issue of inhibition of viral replication by the immune system. On the contrary, replication-competent tumor-specific myxoma virus and vaccinia virus assayed in gliomas implanted in immunocompetent hosts showed improved oncolytic activity when drugs like rapamycin suppressing the innate IFN I responses were coadministered. ${ }^{39,40}$

Here we established a syngeneic immunocompetent murine model of HGG expressing HER2, in which we evaluated the impact of the host immune system on R-LM113 anti-glioma efficacy. $\mathrm{BALB} / \mathrm{c}$ mice were chosen because they are known to be susceptible to HSV infection. ${ }^{18}$

None of R-LM113 intracranially inoculated BALB/C mice developed fatal inflammation nor showed signs of infection (EGFP expression), demonstrating that R-LM113 did not elicit a dangerous immune response and therefore it is safe also in an immunocompetent animal.

The oncolytic virus was clearly able to infect tumor cells in vivo and, notably, infected EGFP-expressing cells were found also in animals dying very late (that is, 160 days from the treatment), a time surely more than sufficient for an antigen to elicit an immune 
response. Interestingly, the EGFP-positive cells were spread in the brain even in location far away from the injection site, demonstrating that the immune system of $B A L B / c$ is not able to limit the spread in the tissue (Figure 4e).

In terms of efficacy, the comparison between the results obtained in the immunocompetent BALB/C mouse strain and those obtained in the immunodeficient NOD/SCID mouse strain, described in our previous report, ${ }^{9}$ shows that the efficacy of R-LM113 is indeed virtually undistinguishable both in early and late treatment experimental schemes.

This study shows that the R-LM113 virus is not spoiled nor made dangerous for the recipient by the reaction of a competent immune system and thus represents a step forward towards the possibility to treat human HGGs with retargeted oHSVs. In perspective, it is also to consider the possibility of improving the efficacy of oncolytic effect by 'arming' retargeted oHSVs with cytokines as IL-12, GM-CSF or IL-21 in order to recruit effector cells and enhance the response of the immune system against the tumor. ${ }^{7,41,42}$

\section{CONFLICT OF INTEREST}

LM serves as Consultant of Catherex Inc., and is coinventor on a patent filed by the University of Bologna covering herpes simplex virus with modified tropism, uses and process of preparation thereof. The remaining authors declare no conflict of interest.

\section{ACKNOWLEDGEMENTS}

This work was supported by GR-2008-1135643 grant from the Italian Ministry of Health to PM and LM, by NUSUG GRANT to PM, by grants from the Department of Experimental Pathology, University of Bologna (Pallotti funds), by the University of Bologna RFO (Ricerca Fondamentale Orientata); and Fondazione S. Paolo to PM (Molecular and cellular basis of glioma). The fellowship of ER was supported by Fondazione Italiana per la Ricerca sul Cancro. VG is the recipient of a fellowship from FoRiBiCA Foundation.

\section{REFERENCES}

1 Stupp R, Hegi ME, Mason WP, van den Bent MJ, Taphoorn MJ, Janzer RC et al. Effects of radiotherapy with concomitant and adjuvant temozolomide versus radiotherapy alone on survival in glioblastoma in a randomised phase III study: 5-year analysis of the EORTC-NCIC trial. Lancet Oncol 2009; 10 459-466.

2 Friedman GK, Pressey JG, Reddy AT, Markert JM, Gillespie GY. Herpes simplex virus oncolytic therapy for pediatric malignancies. Mol Ther 2009; 17: 1125-1135.

3 Campadelli-Fiume G, De Giovanni C, Gatta V, Nanni P, Lollini PL, Menotti L. Rethinking herpes simplex virus: the way to oncolytic agents. Rev Med Virol 2011; 21: 213-226.

4 Menotti L, Campadelli-Fiume G, Nanni P, Lollini PL, De Giovanni C. The Molecular Basis Of Herpesviruses As Oncolytic Agents. Curr Pharm Biotechnol 2011; 13 1795-1803.

5 Geevarghese SK, Geller DA, de Haan HA, Horer M, Knoll AE, Mescheder A et al. Phase I/II study of oncolytic herpes simplex virus NV1020 in patients with extensively pretreated refractory colorectal cancer metastatic to the liver. Hum Gene Ther 2010; 21: 1119-1128.

6 Harrow S, Papanastassiou V, Harland J, Mabbs R, Petty R, Fraser M et al. HSV1716 injection into the brain adjacent to tumour following surgical resection of high-grade glioma: safety data and long-term survival. Gene Ther 2004; 11: 1648-1658.

7 Hu JC, Coffin RS, Davis CJ, Graham NJ, Groves N, Guest PJ et al. A phase I study of OncoVEXGM-CSF, a second-generation oncolytic herpes simplex virus expressing granulocyte macrophage colony-stimulating factor. Clin Cancer Res 2006; 12: 6737-6747.

8 Kaufman HL, Bines SD. OPTIM trial: a phase III trial of an oncolytic herpes virus encoding GM-CSF for unresectable stage III or IV melanoma. Future Oncol 2010; 6 941-949.

9 Gambini E, Reisoli E, Appolloni I, Gatta V, Campadelli-Fiume G, Menotti L et al. Replication-competent herpes simplex virus retargeted to HER2 as therapy for high-grade glioma. Mol Ther 2012; 20: 994-1001.

10 Menotti L, Cerretani A, Hengel H, Campadelli-Fiume G. Construction of a fully retargeted herpes simplex virus 1 recombinant capable of entering cells solely via human epidermal growth factor receptor 2 . J Virol 2008; 82: 10153-10161.
11 Duhem-Tonnelle V, Bieche I, Vacher S, Loyens A, Maurage CA, Collier F et al. Differential distribution of erbB receptors in human glioblastoma multiforme: expression of erbB3 in CD133-positive putative cancer stem cells. J Neuropathol Exp Neurol 2010; 69: 606-622.

12 Nabika S, Kiya K, Satoh H, Mizoue T, Kondo H, Katagiri M et al. Prognostic significance of expression patterns of EGFR family, p21 and p27 in high-grade astrocytoma. Hiroshima J Med Sci 2010; 59: 65-70.

13 Gulati S, Ytterhus B, Granli US, Gulati M, Lydersen S, Torp SH. Overexpression of c-erbB2 is a negative prognostic factor in anaplastic astrocytomas. Diagn Pathol 2010; 5: 18.

14 Hiesiger EM, Hayes RL, Pierz DM, Budzilovich GN. Prognostic relevance of epidermal growth factor receptor (EGF-R) and c-neu/erbB2 expression in glioblastomas (GBMs). J Neurooncol 1993; 16: 93-104.

15 Mineo JF, Bordron A, Baroncini M, Maurage CA, Ramirez C, Siminski RM et al. Low HER2-expressing glioblastomas are more often secondary to anaplastic transformation of low-grade glioma. J Neurooncol 2007; 85: 281-287.

16 Press MF, Cordon-Cardo C, Slamon DJ. Expression of the HER-2/neu proto-oncogene in normal human adult and fetal tissues. Oncogene 1990; 5: 953-962.

17 Lopez C. Resistance to herpes simplex virus type 1 (HSV-1). Curr Top Microbiol Immunol 1981; 92: 15-24.

18 Lopez C. Genetics of natural resistance to herpesvirus infections in mice. Nature 1975; 258: 152-153.

19 Appolloni I, Calzolari F, Tutucci E, Caviglia S, Terrile M, Corte G et al. PDGF-B induces a homogeneous class of oligodendrogliomas from embryonic neural progenitors. Int J Cancer 2009; 124: 2251-2259.

20 Appolloni I, Calzolari F, Corte G, Perris R, Malatesta P. Six3 controls the neural progenitor status in the murine CNS. Cerebral cortex 2008; 18: 553-562.

21 Appolloni I, Calzolari F, Barilari M, Terrile M, Daga A, Malatesta P. Antagonistic modulation of gliomagenesis by Pax6 and Olig2 in PDGF-induced oligodendroglioma. Int J Cancer 2012; 131: E1078-E1087.

22 Terrile M, Appolloni I, Calzolari F, Perris R, Tutucci E, Malatesta P. PDGF-B-driven gliomagenesis can occur in the absence of the proteoglycan NG2. BMC Cancer 2010; 10: 550.

23 Casalini P, Botta L, Menard S. Role of p53 in HER2-induced proliferation or apoptosis. J Biol Chem 2001; 276: 12449-12453.

24 Lundberg P, Cantin E. A potential role for CXCR3 chemokines in the response to ocular HSV infection. Curr Eye Res 2003; 26: 137-150.

25 Halford WP, Balliet JW, Gebhardt BM. Re-evaluating natural resistance to herpes simplex virus type 1. J Virol 2004; 78: 10086-10095.

26 Lopez C. Resistance to HSV-1 in the mouse is governed by two major, independently segregating, non-H-2 loci. Immunogenetics 1980; 11: 87-92.

27 Calzolari F, Appolloni I, Tutucci E, Caviglia S, Terrile M, Corte G et al. Tumor progression and oncogene addiction in a PDGF-B-induced model of gliomagenesis. Neoplasia (New York, NY) 2008; 10: 1373-1382.

28 Dai C, Celestino JC, Okada Y, Louis DN, Fuller GN, Holland EC. PDGF autocrine stimulation dedifferentiates cultured astrocytes and induces oligodendrogliomas and oligoastrocytomas from neural progenitors and astrocytes in vivo. Genes Dev 2001; 15: 1913-1925.

29 Russell SJ, Peng KW, Bell JC. Oncolytic virotherapy. Nat Biotechnol 2012; 30: 658-670.

30 Lasaro MO, Ertl HC. New insights on adenovirus as vaccine vectors. Mol Ther 2009; 17: $1333-1339$

31 Yang Y, Li Q, Ertl HC, Wilson JM. Cellular and humoral immune responses to viral antigens create barriers to lung-directed gene therapy with recombinant adenoviruses. J Virol 1995; 69: 2004-2015.

32 Todo T, Rabkin SD, Sundaresan P, Wu A, Meehan KR, Herscowitz HB et al. Systemic antitumor immunity in experimental brain tumor therapy using a multimutated, replication-competent herpes simplex virus. Hum Gene Ther 1999; 10: $2741-2755$.

33 Kaufman HL, Kim DW, DeRaffele G, Mitcham J, Coffin RS, Kim-Schulze S. Local and distant immunity induced by intralesional vaccination with an oncolytic herpes virus encoding GM-CSF in patients with stage IIIc and IV melanoma. Ann Surg Oncol 2010; 17: 718-730.

34 Hellums EK, Markert JM, Parker JN, He B, Perbal B, Roizman B et al. Increased efficacy of an interleukin-12-secreting herpes simplex virus in a syngeneic intracranial murine glioma model. Neuro Oncol 2005; 7: 213-224.

35 Parker JN, Gillespie GY, Love CE, Randall S, Whitley RJ, Markert JM. Engineered herpes simplex virus expressing IL-12 in the treatment of experimental murine brain tumors. Proc Nat Acad Sci USA 2000; 97: 2208-2213.

36 Castro MG, Candolfi M, Kroeger K, King GD, Curtin JF, Yagiz K et al. Gene therapy and targeted toxins for glioma. Curr Gene Ther 2011; 11: 155-180.

37 Curtin JF, Liu N, Candolfi M, Xiong W, Assi H, Yagiz K et al. HMGB1 mediates endogenous TLR2 activation and brain tumor regression. PLoS Med 2009; 6: e10. 
38 Ali S, Curtin JF, Zirger JM, Xiong W, King GD, Barcia C et al. Inflammatory and antiglioma effects of an adenovirus expressing human soluble Fms-like tyrosine kinase 3 ligand (hsFlt3L): treatment with hsFlt3L inhibits intracranial glioma progression. Mol Ther 2004; 10: 1071-1084.

39 Lun X, Chan J, Zhou H, Sun B, Kelly JJ, Stechishin OO et al. Efficacy and safety/ toxicity study of recombinant vaccinia virus JX-594 in two immunocompetent animal models of glioma. Mol Ther 2010; 18: 1927-1936.

40 Lun X, Alain T, Zemp FJ, Zhou H, Rahman MM, Hamilton MG et al. Myxoma virus virotherapy for glioma in immunocompetent animal models: optimizing

Supplementary Information accompanies the paper on Cancer Gene Therapy website (http://www.nature.com/cgt)

administration routes and synergy with rapamycin. Cancer Res 2010; 70: 598-608.

41 Daga A, Orengo AM, Gangemi RM, Marubbi D, Perera M, Comes A et al. Glioma immunotherapy by IL-21 gene-modified cells or by recombinant IL-21 involves antibody responses. International journal of cancer. Int $J$ Cancer 2007; 121: 1756-1763.

42 Andreansky S, He B, van Cott J, McGhee J, Markert JM, Gillespie GY et al. Treatment of intracranial gliomas in immunocompetent mice using herpes simplex viruses that express murine interleukins. Gene Ther 1998; 5: 121-130. 